\title{
Communication Logics for Networked Control Systems
}

\author{
Yonggang $\mathrm{Xu}$
}

\begin{abstract}
This paper addresses the control of spatially distributed processes. We utilize a distributed architecture in which multiple local controllers coordinate their efforts through a data network that allows information exchange. We focus our work on linear time invariant processes disturbed by Gaussian white noise and propose several logics to determine when the local controllers should communicate. We provide conditions under which these logics guarantee boundedness and investigate the trade-off between the amount of information exchanged and the performance achieved. The resulting closed-loop systems evolve according to stochastic differential equations with resets triggered by stochastic counters. This type of stochastic hybrid system seems to be interesting on its own. The theoretical results are validated through Monte Carlo simulations.
\end{abstract}

\section{INTRODUCTION}

The architectures for feedback control of spatially distributed processes generally fall in one of the three classes centralized, decentralized, and distributed. Centralized architectures yield the best performance because they pose the least constraints on the structure of the controller, whereas decentralized architectures are the simplest to implement. We pursue here distributed architectures, as they provide a range of compromise solutions between the two extremes. We assume that the communication among local controllers is supported by a data network that allows information between local controllers to be exchanged at discrete time instants.

This paper aims at understanding the trade-off between the amount of information exchanged and the performance achieved. A significant deal of work exists on how to reduce communication in networked control systems. The problem of stabilization with finite communication bandwidth was introduced by [2], [3] and further pursued by [4]-[8]. [2] investigated an estimation problem under the constraint that observations must be coded digitally and transmitted over a channel with finite capacity. In [3] they addressed the corresponding stabilization problem under similar limitations. [4], [5] and [8] determined the minimum bandwidth (measured in discrete symbols per second) needed to stabilize a linear process. In all these references a digital communication channel was assumed so that any information transmitted had to be quantized.

We depart from the work summarized above in that we only penalize the number of times that information is exchanged. This is motivated by the fact that in the most widely used communication protocols, such as Asynchronous Transfer Mode (ATM) and Ethernet, there is a fixed overhead per data packet.

\footnotetext{
This research was supported by the National Science Foundation under the grants: CCR-0311084 and ECS-0093762.

The authors are with Dept. of Electrical and Computer Eng., Univ. of California, Santa Barbara, CA 93106
}

\author{
João P. Hespanha
}

This paper is focused on spatially distributed processes whose dynamics are decoupled but for which the control objective is not, e.g., the control of a group of aircraft to fly in a geometric formation (far enough from each other so that their dynamics are decoupled). However, many of these ideas could be extended to coupled processes.

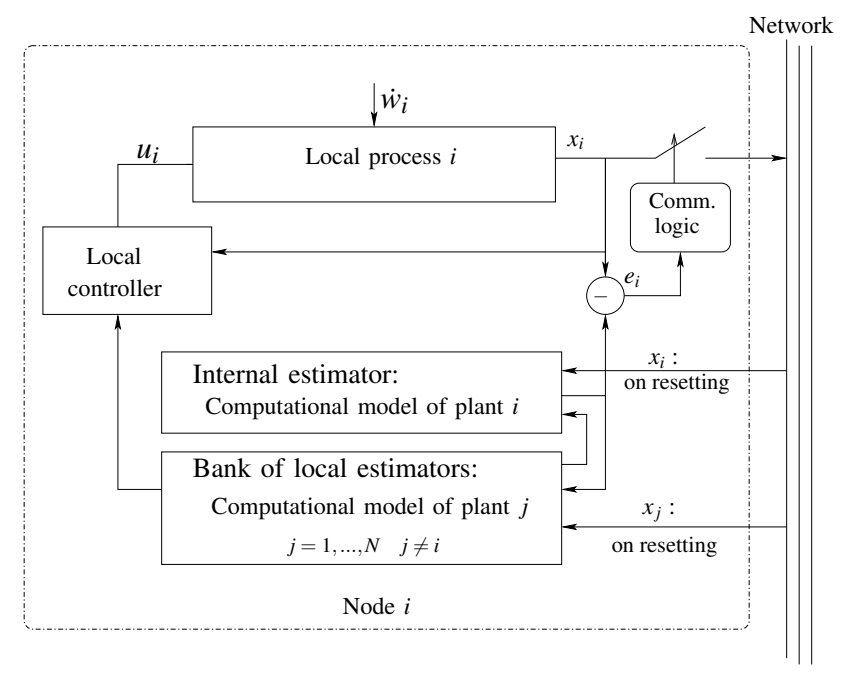

Fig. 1. One of the nodes in a networked control system.

We view each process with an associated local controller as a node. The overall control system consists of $N$ nodes connected via a data network. Fig. 1 depicts the internal structure of the $i$ th node. Each node consists of a local process, a local controller, a bank of local estimators that estimates the state of the remaining $N-1$ processes, and a communication logic that schedules when to transmit data to the other nodes. The communication logic makes use of an internal estimator to determine how well other nodes can "predict" the state of the local process.

The bank of local estimators are simply models of the other local processes. They run open-loop most of the time but are sometimes reset to "correct" values received through the network. These resets do not necessarily occur periodically. It is the responsibility of each node to broadcast to the network the state of its local process when it believes that the open-loop estimates kept by the remaining nodes have significantly deviated from the true value. To this effect it also constructs an internal open-loop estimator of the state of its local process and uses it to decide when to broadcast. This type of architecture was proposed by Yook, Tilbury, and Soparkar [9] for the control of discrete-time distributed systems.

Several algorithms can be used by the communication logic to determine when the state of the local process 
should be broadcast. The quality of a communication logic should be judged in terms of the control performance it can achieve for a given rate of message broadcasts. One simple algorithm would consist of broadcasting messages periodically. However, as we shall see, this is not optimal because data may be transmitted with little new information. [9] proposed that a node should broadcast the true value of the state of the local process when it differs from the estimate known to the remaining nodes by more than a given threshold. For the linear discrete-time case, they showed that this scheme results in a system that is BIBO stable. The relation between the threshold level and the message exchange rate was investigated through simulation in the context of examples.

We proposed new communication logics that can be analyzed to determine stability as well as the trade-off between communication (in terms of average message exchange rates) and performance. We start by considering stochastic communication logics for which the probability of a node broadcasting a message is a function of the current estimation error. Deterministic communication logics similar to the ones proposed in [9] are also considered. We will see that the latter can be viewed as limiting cases of the former.

The stochastic communication logics are based on doubly stochastic Poisson processes (DSPPs) [10]. In essence, the state of the local process is broadcast according to a Poisson process whose rate depends on the estimation error. This type of stochastic hybrid system seems to be interesting on its own. For stochastic communication logics, our analysis uses tools from jump diffusion processes. Deterministic logics are analyzed by considering first exit time problems.

In Section II, the control-communication architecture is formally described for the case of two linear time-invariant processes. Equations for the estimation errors and the communication rate are derived. Stochastic communication logics are analyzed in Section III, whereas deterministic ones are addressed in Section IV. Simulation results are presented in Section $\mathrm{V}$ for a second order leader-follower problem. We also provide trade-off curves showing the average communication rate versus the variance of the estimation error for an unstable process. Section VI contains conclusions and directions for future work.

\section{NETWORKED CONTROL SYSTEM MODEL}

For simplicity, we consider only two nodes like the ones in Fig. 1. The processes are assumed linear time-invariant with an exogenous disturbance input,

$$
\dot{\mathbf{x}}_{i}=A_{i} \mathbf{x}_{i}+B_{i} \mathbf{u}_{i}+\sigma_{i} \dot{\mathbf{w}}_{i} \quad \forall i \in\{1,2\},
$$

where $\mathbf{x}_{i} \in \mathbb{R}^{n_{i}}$ denotes the state, $\mathbf{u}_{i} \in \mathbb{R}^{m_{i}}$ the control input, and $\dot{\mathbf{w}}_{i} \ell_{i}$-dimensional standard Gaussian white noise. The two noise processes are assumed independent and all matrices are real and of appropriate dimensions. It is assumed that all the states can be measured. Otherwise state observers should be used.

We assume given state-feedback control laws

$$
\mathbf{u}_{i}=K_{i 1} \mathbf{x}_{1}+K_{i 2} \mathbf{x}_{2}, \quad \forall i \in\{1,2\}
$$

that would provide adequate performance in a centralized configuration, i.e., if the states of both processes were available to both local controllers. In a centralized configuration, the closed-loop system would be

$$
\begin{aligned}
& \dot{\mathbf{x}}_{1}=\left(A_{1}+B_{1} K_{11}\right) \mathbf{x}_{1}+B_{1} K_{12} \mathbf{x}_{2}+\sigma_{1} \dot{\mathbf{w}}_{1} \\
& \dot{\mathbf{x}}_{2}=\left(A_{2}+B_{2} K_{22}\right) \mathbf{x}_{2}+B_{2} K_{21} \mathbf{x}_{1}+\sigma_{2} \dot{\mathbf{w}}_{2} .
\end{aligned}
$$

Since the state of the $i$ th process is not directly available at the $j$ th node $(j \neq i, i, j \in\{1,2\})$, we build at the node $j$ an unbiased estimate $\hat{\mathbf{x}}_{i}$ of the real state $\mathbf{x}_{i}$. This is done using the open-loop "computational models,"

$$
\begin{aligned}
& \dot{\hat{\mathbf{x}}}_{1}=\left(A_{1}+B_{1} K_{11}\right) \hat{\mathbf{x}}_{1}+B_{1} K_{12} \hat{\mathbf{x}}_{2} \\
& \dot{\hat{\mathbf{x}}}_{2}=\left(A_{2}+B_{2} K_{22}\right) \hat{\mathbf{x}}_{2}+B_{2} K_{21} \hat{\mathbf{x}}_{1} .
\end{aligned}
$$

Both nodes compute both estimates: not only the estimate of the state of the other process, but also the estimate of its own local state. The latter is used by the communication logic to monitor the quality of the estimate that the other node is currently using. In the distributed architecture, the centralized laws (1) are replaced by

$$
\begin{aligned}
& \mathbf{u}_{1}=K_{11} \mathbf{x}_{1}+K_{12} \hat{\mathbf{x}}_{2} \\
& \mathbf{u}_{2}=K_{21} \hat{\mathbf{x}}_{1}+K_{22} \mathbf{x}_{2} .
\end{aligned}
$$

The distributed control laws (4) result in a closed-loop dynamics given by

$$
\begin{aligned}
& \dot{\mathbf{x}}_{1}=\left(A_{1}+B_{1} K_{11}\right) \mathbf{x}_{1}+B_{1} K_{12} \hat{\mathbf{x}}_{2}+\sigma_{1} \dot{\mathbf{w}}_{1} \\
& \dot{\mathbf{x}}_{2}=\left(A_{2}+B_{2} K_{22}\right) \mathbf{x}_{2}+B_{2} K_{21} \hat{\mathbf{x}}_{1}+\sigma_{2} \dot{\mathbf{w}}_{2},
\end{aligned}
$$

to be contrasted with (2). From (3) and (5), the dynamics of the estimation errors $\mathbf{e}_{i}:=\hat{\mathbf{x}}_{i}-\mathbf{x}_{i}, i \in\{1,2\}$, are given by

$$
\begin{aligned}
\dot{\mathbf{e}}_{1} & =\left(A_{1}+B_{1} K_{11}\right) \mathbf{e}_{1}-\sigma_{1} \dot{\mathbf{w}}_{1} \\
\dot{\mathbf{e}}_{2} & =\left(A_{2}+B_{2} K_{22}\right) \mathbf{e}_{2}-\sigma_{2} \dot{\mathbf{w}}_{2} .
\end{aligned}
$$

However, these equations do not hold all the time. Let us denote by $0=: \mathbf{t}_{0}<\mathbf{t}_{1}<\mathbf{t}_{2}<\cdots$ the times at which the communication logic at node 1 broadcasts its local process state $\mathbf{x}_{1}$. If one were to neglect quantization errors and network delays, both nodes would incorporate this information into their estimates at time $\mathbf{t}_{k}$ and

$$
\mathbf{e}_{1}\left(\mathbf{t}_{k}\right)=0, \quad \forall k
$$

The equation (6) then holds between consecutive instants $\mathbf{t}_{k}$. If the decision to broadcast $\mathbf{x}_{1}$ is only based on the current value of $\mathbf{e}_{1}$, the estimation errors are independent of each other and $\mathbf{e}_{i}$ is also independent of $\dot{\mathbf{w}}_{j}$ for $i \neq j$.

The effect of quantization error can be viewed as resetting $\mathbf{e}_{1}$ to a non-zero value $\mathbf{z}_{k}$, i.e.,

$$
\mathbf{e}_{1}\left(\mathbf{t}_{k}\right)=\mathbf{z}_{k}, \quad \forall k,
$$

and the distribution of $\mathbf{z}_{k}$ is characterized by the quantization mechanism. Typically, it is uniform over $\left[-\frac{q}{2}, \frac{q}{2}\right]$, where $q$ is the quantization level. The analysis of network delay is more involved and is left for future research. 
To understand the effect of the distributed architecture on the performance of the closed-loop system, we write the closed-loop dynamics (5) in terms of the estimation errors:

$$
\begin{aligned}
& \dot{\mathbf{x}}_{1}=\left(A_{1}+B_{1} K_{11}\right) \mathbf{x}_{1}+B_{1} K_{12} \mathbf{x}_{2}+\sigma_{1} \dot{\mathbf{w}}_{1}+B_{1} K_{12} \mathbf{e}_{2}, \\
& \dot{\mathbf{x}}_{2}=\left(A_{2}+B_{2} K_{22}\right) \mathbf{x}_{2}+B_{2} K_{21} \mathbf{x}_{1}+\sigma_{2} \dot{\mathbf{w}}_{2}+B_{2} K_{21} \mathbf{e}_{1} .
\end{aligned}
$$

Comparing these equations with (2), we observe that the penalty paid for a distributed architecture is the additive "disturbance" terms $B_{i} K_{i j} \mathbf{e}_{j}$. Therefore, the performance of the closed-loop distributed architecture is to a great extent related to the properties of the errors generated by the equations (6) and (8).

We study the dynamics of the estimation error $\mathbf{e}(t)$. To simplify notations, (6) and (8) are written in the following generic form,

$$
\dot{\mathbf{e}}=A \mathbf{e}+\sigma \dot{\mathbf{w}}, \quad \mathbf{e}\left(\mathbf{t}_{k}\right)=\mathbf{z}_{k},
$$

where $\mathbf{e} \in \mathbb{R}^{n}, A \in \mathbb{R}^{n \times n}, \sigma \in \mathbb{R}^{n \times \ell}$, and $\dot{\mathbf{w}}$ is $\ell$-dimensional standard Gaussian white noise. At discrete time instants $0=: \mathbf{t}_{0}<\mathbf{t}_{1}<\cdots, \mathbf{e}\left(\mathbf{t}_{k}\right)$ jumps to $\mathbf{z}_{k}$. We assume that all $\mathbf{z}_{k}$ are i.i.d., with zero-mean and probability distribution $\mu(z)$. It is convenient to write (9) in a jump diffusion form,

$$
d \mathbf{e}=A \mathbf{e} d t+\sigma d \mathbf{w}+d \mathbf{J}
$$

where $d \mathbf{J}(t)$ is a jump term that is zero almost everywhere, except at discrete time instants $\mathbf{t}_{k}, k \geq 0$, when it is given by

$$
d \mathbf{J}\left(\mathbf{t}_{k}\right):=\lim _{t \downarrow \mathbf{t}_{k}} \mathbf{e}(t)-\lim _{t \uparrow \mathbf{t}_{k}} \mathbf{e}(t)=\mathbf{z}_{k}-\lim _{t \uparrow \mathbf{t}_{k}} \mathbf{e}(t)
$$

We measure the "communication cost" of a particular communication logic in terms of the communication rate, defined to be the asymptotic rate that messages are sent, i.e.,

$$
R:=\lim _{k \rightarrow \infty} \mathrm{E}\left[\frac{k}{\mathbf{t}_{k}}\right] .
$$

Defining $\mathbf{T}_{k}:=\mathbf{t}_{k}-\mathbf{t}_{k-1}$ to be the intercommunication time between the $(k-1)$ th and the $k$ th messages, since all the $\mathbf{T}_{k}$ are i.i.d., it is straightforward to show that

$$
R=\lim _{k \rightarrow \infty} \mathrm{E}\left[\frac{k}{\sum_{i=1}^{k} \mathbf{T}_{k}}\right]=\frac{1}{\mathrm{E}\left[\mathbf{T}_{k}\right]} .
$$

This paper investigates the relation between performance, measured in terms of the statistical moments of the estimation error $\mathbf{e}$ and communication cost, measured in terms of the communication rate $R$, for several communication logics.

\section{STOCHASTIC COMMUNICATION LOGICS}

The idea behind stochastic communication logics is for each node to broadcast at an average rate that depends on the current value of the estimation error. To formalize this we consider a DSPP $\mathbf{N}(t)$, which is an integer-valued stochastic process whose increments are associated with message exchanges. In particular, $\mathbf{N}(t)$ is constant except at the times $\mathbf{t}_{k}$, where it increases by one. The jumps of the random process $\mathbf{e}$ in (10) are synchronized with the increments of $\mathbf{N}(t)$ and therefore we can re-write (11) as

$$
d \mathbf{J}(t)=\left(\mathbf{z}_{k}-\mathbf{e}(t)\right) d \mathbf{N}(t),
$$

where $d \mathbf{N}(t):=\lim _{\tau \downarrow t} \mathbf{N}(\tau)-\lim _{\tau \uparrow t} \mathbf{N}(t)$.

The instantaneous rate at which increments occur is a function of the estimation error $\mathbf{e}(t)$. In particular we take $\mathbf{N}(t)$ to be a DSPP with intensity $\lambda(\mathbf{e})$, which has the property that

$$
\mathrm{E}[\mathbf{N}(t)-\mathbf{N}(\tau)]=\mathrm{E}\left[\int_{\tau}^{t} \lambda(\mathbf{e}(s)) d s\right], \quad \forall t \geq \tau \geq 0
$$

where $\lambda: \mathbb{R}^{n} \rightarrow[0, \infty)$ is an intensity function. The communication rate $R$ is then given by

$$
R:=\lim _{t \rightarrow \infty} \frac{\mathrm{E}[\mathbf{N}(t)-\mathbf{N}(0)]}{t}=\lim _{t \rightarrow \infty} \frac{\int_{0}^{t} \mathrm{E}[\lambda(\mathbf{e}(s))] d s}{t},
$$

which shows that when $\mathrm{E}[\lambda(\mathbf{e}(s))]$ converges as $s \rightarrow \infty$, the limit is the communication rate $R$.

Solutions to (10), (13) are defined in the Itô sense [11], [12]. But for the purpose of stability analysis, it is sufficient to consider its generator [13]. Given a twice continuously differentiable function $f$ defined on $\mathbb{R}^{n}$ and a jump diffusion process $\mathbf{e}$, the generator $\mathscr{L}$ of $\mathbf{e}$ is defined by

$(\mathscr{L} f)(e):=\lim _{\tau \rightarrow t} \frac{\mathrm{E}^{e}[f(\mathbf{e}(\tau)]-f(e)}{\tau-t}, \quad \forall e \in \mathbb{R}^{n}, \tau>t \geq 0$,

where $\mathrm{E}^{e}[f(\mathbf{e}(\tau)]$ denotes the expectation of $f(\mathbf{e}(\tau))$ given $\mathbf{e}(t)=e$. It can be shown that the generator for the jump diffusion process described by (10), (13) is given by

$$
\begin{aligned}
\mathscr{L} f(e)= & \frac{\partial f(e)}{\partial e} \cdot A e+\frac{1}{2} \operatorname{trace}\left[\sigma^{\prime} \frac{\partial^{2} f(e)}{\partial e^{2}} \sigma\right] \\
& +\lambda(e)\left(\int f(z) d \mu(z)-f(e)\right),
\end{aligned}
$$

where $\frac{\partial f(e)}{\partial e}$ and $\frac{\partial^{2} f(e)}{\partial e^{2}}$ denote the gradient vector and Hessian matrix of $f$ respectively [12].

Setting $e=\mathbf{e}(t)$ in (15) and taking expectation, one obtains

$$
\frac{\mathrm{d}}{\mathrm{d} t} \mathrm{E}[f(\mathbf{e}(t))]=\mathrm{E}[(\mathscr{L} f)(\mathbf{e}(t))],
$$

from which stability properties of the process $\mathbf{e}(t)$ can be deduced by appropriate choices of $f(\cdot)$.

\section{A. Constant rate}

We start by considering a constant intensity $\lambda(e)=\gamma$ for the DSPP. From (14), the corresponding communication rate is $R=\gamma$. Due to space limitations we do not include the proof of the following results [14].

Theorem 1: Let $\mathbf{e}$ be the jump diffusion process defined by (10) and (13) with $\lambda(e)=\gamma, \forall e$.

1) When $\gamma>\max \{\mathfrak{R}[e i g(A)]\}, \mathrm{E}[\mathbf{e}(t)]$ converges to zero exponentially fast.

2) When $\gamma>2 m \max \{\Re[\operatorname{eig}(A)]\}$, for any $m \geq 1$, $\mathrm{E}\left[(\mathbf{e}(t) \cdot \mathbf{e}(t))^{m}\right]$ is bounded. 
3) When $\gamma>2 \max \{\Re[\operatorname{eig}(A)]\}$, and $P, Q$ are $n \times n$ positive definite matrices and $c$ a positive constant such that

$$
P\left(A-\frac{\gamma}{2} I\right)+\left(A-\frac{\gamma}{2} I\right)^{\prime} P \leq-Q, \quad Q \geq c P,
$$

then $\mathrm{E}[\mathbf{e}(t) \cdot \mathbf{e}(t)]$ is uniformly bounded and

$$
\lim _{t \rightarrow \infty} \mathrm{E}[\mathbf{e}(t) \cdot P \mathbf{e}(t)] \leq \frac{\gamma \rho^{2}+\theta}{c},
$$

where $\rho^{2}:=\int z \cdot P z d \mu(z)$, and $\theta:=\operatorname{trace}\left(\sigma^{\prime} P \sigma\right)$.

\section{B. Error-dependent rate}

We now consider an intensity for the DSPP that depends on the current estimation error. The rationale is that a larger estimation error should more rapidly lead to a message exchange. We consider intensities of the form

$$
\lambda(e)=(e \cdot P e)^{k}, \quad \forall e,
$$

where $P$ is some positive definite matrix and $k$ a positive integer.

Theorem 2: Let e be the jump diffusion process defined by (10) and (13) with intensity (19). The communication rate and all the finite moments of $\mathbf{e}(t)$ are bounded for all $k>0$.

To prove this theorem, we need the following Lemma (proved in the Appendix), which relates the expectations of different moments of a positive random variable.

Lemma 1: Given a scalar random variable $\mathbf{x}$ that is nonnegative with probability one, a positive constant $\delta$, and positive integers $k>\ell>0$, we have that $\mathrm{E}\left[\mathbf{x}^{k}\right] \geq$ $\delta^{\ell} \mathrm{E}\left[\mathbf{x}^{k-\ell}\right]-\delta^{k}$.

Proof: [Theorem 2] Choose $c_{1}$ sufficiently large so that $A-\frac{c_{1}}{2} I$ is asymptotically stable. Then there exists a matrix $P>0$ such that

$$
P\left(A-\frac{c_{1}}{2} I\right)+\left(A-\frac{c_{1}}{2} I\right) P<0,
$$

i.e., $P A+A^{\prime} P<c_{1} P$. Moreover $P \sigma \sigma^{\prime} P \leq c_{2} P$ for sufficiently large $c_{2}>0$.

We start by proving that the $m$ th moment of $\mathbf{e}(t)$ is bounded when $m>k$. Define $V(e):=(e \cdot P e)^{m}$. From (16)

$$
\begin{aligned}
& \mathscr{L} V(e) \\
= & m(e \cdot P e)^{m-1} e \cdot\left(P A+A^{\prime} P\right) e+\lambda(e) \rho^{2 m}-\lambda(e) V(e) \\
+ & 2 m(m-1)(e \cdot P e)^{m-2} e \cdot P \sigma \sigma^{\prime} P e+m(e \cdot P e)^{m-1} \theta \\
= & m(e \cdot P e)^{m-1} e \cdot\left(P A+A^{\prime} P\right) e+\rho^{2 m}(e \cdot P e)^{k}-(e \cdot P e)^{m+k} \\
+ & 2 m(m-1)(e \cdot P e)^{m-2} e \cdot P \sigma \sigma^{\prime} P e+m(e \cdot P e)^{m-1} \theta \\
\leq & c_{1} m(e \cdot P e)^{m}+\rho^{2 m}(e \cdot P e)^{k}-(e \cdot P e)^{m+k} \\
+ & m\left(2 c_{2}(m-1)+\theta\right)(e \cdot P e)^{m-1} .
\end{aligned}
$$

From this and (17), we conclude that

$$
\begin{aligned}
\frac{\mathrm{d}}{\mathrm{d} t} \mathrm{E}[V(\mathbf{e})] & \leq c_{1} m \mathrm{E}[V(\mathbf{e})]+\rho^{2 m} \mathrm{E}\left[(\mathbf{e} \cdot P \mathbf{e})^{k}\right]-\mathrm{E}\left[(\mathbf{e} \cdot P \mathbf{e})^{m+k}\right] \\
& +m\left(2 c_{2}(m-1)+\theta\right) \mathrm{E}\left[(\mathbf{e} \cdot P \mathbf{e})^{m-1}\right] .
\end{aligned}
$$

Given some $\delta_{1}, \delta_{2}, \delta_{3}>0$, we conclude from Lemma 1 that

$$
\begin{aligned}
\mathrm{E}\left[(\mathbf{e} \cdot P \mathbf{e})^{k}\right] & \leq \frac{\mathrm{E}[V(\mathbf{e})]}{\delta_{1}^{m-k}}+\delta_{1}^{k}, \\
\mathrm{E}\left[(\mathbf{e} \cdot P \mathbf{e})^{m+k}\right] & \geq \delta_{2}^{k} \mathrm{E}[V(\mathbf{e})]-\delta_{2}^{m+k}, \\
\mathrm{E}\left[(\mathbf{e} \cdot P \mathbf{e})^{m-1}\right] & \leq \frac{1}{\delta_{3}} \mathrm{E}[V(\mathbf{e})]+\delta_{3}^{m-1},
\end{aligned}
$$

therefore

$$
\begin{aligned}
& \quad \frac{\mathrm{d}}{\mathrm{d} t} \mathrm{E}[V(\mathbf{e})] \\
& \leq c_{1} m \mathrm{E}[V(\mathbf{e})]+\rho^{2 m}\left(\frac{\mathrm{E}[V(\mathbf{e})]}{\delta_{1}^{m-k}}+\delta_{1}^{k}\right)-\left(\delta_{2}^{k} \mathrm{E}[V(\mathbf{e})]-\delta_{2}^{m+k}\right) \\
& \quad+m\left(2 c_{2}(m-1)+\theta\right)\left(\frac{\mathrm{E}[V(\mathbf{e})]}{\delta_{3}}+\delta_{3}^{m-1}\right) \\
& \quad\left(c_{1} m+\frac{\rho^{2 m}}{\delta_{1}^{m-k}}-\delta_{2}^{k}+m \frac{2 c_{2}(m-1)+\theta}{\delta_{3}}\right) \mathrm{E}[V(\mathbf{e})] \\
& \quad+\rho^{2 m} \delta_{1}^{k}+\delta_{2}^{m+k}+m\left(2 c_{2}(m-1)+\theta\right) \delta_{3}^{m-1} .
\end{aligned}
$$

For sufficiently large $\delta_{2}$,

$$
c_{1} m+\frac{\rho^{2 m}}{\delta_{1}^{m-k}}-\delta_{2}^{k}+\frac{m\left(2 c_{2}(m-1)+\theta\right)}{\delta_{3}}<0,
$$

and the boundedness of $\mathrm{E}[V(\mathbf{e})]$ and consequently of $\mathrm{E}[(\mathbf{e}$. e) ${ }^{m}$ ] follows.

To prove the boundedness of the $m$ th moment of $\mathbf{e}(t)$ for $m \leq k$, we use Lemma 1 to bound

$$
\mathrm{E}\left[(\mathbf{e} \cdot \mathbf{e})^{m}\right] \leq \frac{\mathrm{E}\left[(\mathbf{e} \cdot \mathbf{e})^{k+1}\right]}{\delta_{4}^{k+1-m}}+\delta_{4}^{m}
$$

where $\delta_{4}>0$. Since the boundedness of the $(k+1)$ th moment has already been established, we conclude that the $m$ th moment is also bounded for $m \leq k$.

\section{Deterministic COMMUNICATION LOGICS}

We now consider communication logics that utilize deterministic rules. We take a continuous positive and radially unbounded communication index $S: \mathbb{R}^{n} \rightarrow \mathbb{R}^{+}$and force a node to broadcast its state when $S(\mathbf{e}) \geq 1$. In particular, a message exchange occurs at time $\mathbf{t}_{k}$ when $\lim _{t \uparrow t_{k}} S(\mathbf{e}(t)) \geq 1$. To avoid chattering, the post-reset value $\mathbf{z}_{k}$ should satisfy $S\left(\mathbf{z}_{k}\right)<1$ with probability one. This type of resetting guarantees that $\mathbf{e}(t)$ is bounded, since

$$
\mathbf{e}(t) \in \mathscr{D}:=\left\{e \in \mathbb{R}^{n} \mid S(e) \leq 1\right\}, \quad \forall t \geq 0,
$$

with probability one.

To determine the communication rate, suppose that a message exchange occurred at time $\mathbf{t}_{k-1}$ and $\mathbf{e}\left(\mathbf{t}_{k-1}\right)$ was reset to $\mathbf{z}_{k-1}$. From $\mathbf{t}_{k-1}$ to the next reset time $\mathbf{t}_{k}, \mathbf{e}(t)$ is a pure diffusion process

$$
\dot{\mathbf{e}}=A \mathbf{e}+\sigma \dot{\mathbf{w}} .
$$

For a given realization $z$ of $\mathbf{z}_{k-1}$, we define $\mathbf{T}_{k}(z)$ to be the inter-communication time if $\mathbf{z}_{k-1}$ was equal to $z$, i.e.,

$$
\mathbf{T}_{k}(z)=\inf \left\{t-\mathbf{t}_{k-1} \geq 0: \mathbf{e}(t) \in \partial \mathscr{D}, \mathbf{e}\left(\mathbf{t}_{k-1}\right)=z\right\},
$$


where $\mathbf{e}(t)$ is governed by (22) for $t \geq \mathbf{t}_{k-1}$ and $\partial \mathscr{D}$ denotes the boundary of $\mathscr{D}$. The random variable $\mathbf{T}_{k}(z)$ is called the first exit time of $\mathbf{e}(t)$ from $\mathscr{D}$. It is in general not easy to obtain the distribution of $\mathbf{T}_{k}(z)$ in closed form, but its expected value can be obtained from Dynkin's equation. In particular, defining $g(z):=\mathrm{E}\left[\mathbf{T}_{k}(z)\right]$, it is known that $g(z)$ is a solution to the following boundary value problem:

$$
\begin{aligned}
& \frac{\partial g(z)}{\partial z} \cdot A z+\frac{1}{2} \operatorname{trace}\left[\sigma^{\prime} \frac{\partial^{2} g(z)}{\partial z^{2}} \sigma\right]=-1, \\
& \forall z \in \mathscr{D}, \quad g(z)=0, \quad \forall z \in \partial \mathscr{D},
\end{aligned}
$$

where $\frac{\partial g(z)}{\partial z}$ and $\frac{\partial^{2} g(z)}{\partial z^{2}}$ denote the gradient vector and Hessian matrix of $g$ respectively [15]. Once $g(z)$ is known, the expected intercommunication time $\mathbf{T}_{k}$ can be obtained from

$$
\mathrm{E}\left[\mathbf{T}_{k}\right]=\mathrm{E}\left[g\left(\mathbf{z}_{k-1}\right)\right]=\int g(z) d \mu(z),
$$

and the communication rate follows from (12)

$$
R=\frac{1}{\int g(z) d \mu(z)} .
$$

In practice, (23) needs to be solved numerically. Since $\mathscr{D}$ is compact, (21) provides an upper bound on $\mathbf{e}(t)$ and consequently on its statistical moments. To obtain tighter bounds one can use Kolmogorov's forward equation with appropriate boundary conditions to compute the probability density function of the error $\mathbf{e}(t)$. However, this method is computationally intensive for higher-order systems.

\section{Simulation RESUlts}

In this section we validate the theoretical results through Monte Carlo simulations. All the simulations are done in Matlab/Simulink. The DSPP $\mathbf{N}(t)$ in (13) is realized by a single binomial test. Specifically, for a fixed time step $h$, a message exchange is triggered at time $t:=k h, k \in \mathbb{N}$, if a binomial test characterized by a probability of success $p=1-e^{-h \lambda(\mathbf{e}(t))}$ succeeds. Convergence results for similar procedures can be found in [16] and references therein.

\section{A. Leader-follower}

A leader-follower problem is used to illustrate the distributed control architecture with different communication logics. The two processes have identical dynamics and are disturbed by uncorrelated white Gaussian noises. The dynamics of the leading and following vehicles are given by

$$
\begin{array}{ll}
\text { leader: } & \dot{\mathbf{x}}_{1}(t)=A \mathbf{x}_{1}(t)+\sigma B \dot{\mathbf{w}}_{1}(t) \\
\text { follower: } & \dot{\mathbf{x}}_{2}(t)=A \mathbf{x}_{2}(t)+B u_{2}+\sigma B \dot{\mathbf{w}}_{2}(t),
\end{array}
$$

where each state $\mathbf{x}_{i}$ contains the position and velocity of one of the vehicles, $u_{2}$ is the follower's control, each $\dot{\mathbf{w}}_{i}(t)$ is standard Gaussian white noise, and $A=\left[\begin{array}{cc}0 & 1 \\ 0 & -0.5\end{array}\right], \sigma=1$, $B=\left[\begin{array}{ll}0 & 1\end{array}\right]^{\prime}$. The follower's control objective is to follow the leader's position. For simplicity, we omitted from (24) exogenous references.
The open-loop state estimator for the leader's state is given by

$$
\dot{\hat{\mathbf{x}}}_{1}=A \hat{\mathbf{x}}_{1}, \quad \hat{\mathbf{x}}_{1}\left(\mathbf{t}_{k}\right)=\mathbf{x}\left(\mathbf{t}_{k}\right)+\mathbf{z}_{k},
$$

where the $\mathbf{t}_{k}$ denote the times at which the leader broadcasts its state $\mathbf{x}\left(\mathbf{t}_{k}\right)$ to the follower, and the $\mathbf{z}_{k}$ are zero-mean uniformly distributed random vectors over an interval of about $5 \%$ of the maximum value of the process state. We use the following linear controller for the follower

$$
u_{2}=-K\left(\mathbf{x}_{2}-\hat{\mathbf{x}}_{1}\right),
$$

with $K=\left[\begin{array}{ll}3.2 & 9.8\end{array}\right]$ obtained from an LQR design.

Tab. I summarizes the communication rates and the variances of both the estimation and the tracking errors for three communication logics: periodic, DSPP with quadratic intensity $\lambda(e)$, and deterministic with a quadratic communication index $S(e)$. To simplify the comparison, the parameters were selected to achieve communication rates approximately equal to one for all logics. We see that both the deterministic and DSPP logics significantly outperform periodic communication.

\section{B. Rate-variance curves}

To study the trade-off between communication rate and estimation error variance, we consider the remote state estimator of a first order unstable process $\dot{\mathbf{x}}=\mathbf{x}+\dot{\mathbf{w}}$. This corresponds to a jump diffusion process defined by (10) and (13) with $A=1, \sigma=1$. The results presented refer to a simulation time of $1000 \mathrm{sec}$.

Fig. 2 depicts the trade-off between communication rate and the variance of the estimation error for four different communication logics: periodic, DSPP with constant intensity, DSPP with quadratic intensity, and deterministic with quadratic communication index. The curves were obtained by varying the parameters that define these logics. For a given communication rate, the DSPP logic with constant intensity results in the largest error, whereas the deterministic logic results in the smallest one. The communication rate obtained with the DSPP logic for the quadratic $\lambda(e)$ is significantly smaller than the upper bound provided by (20), which for this example is numerically equal to 1 .

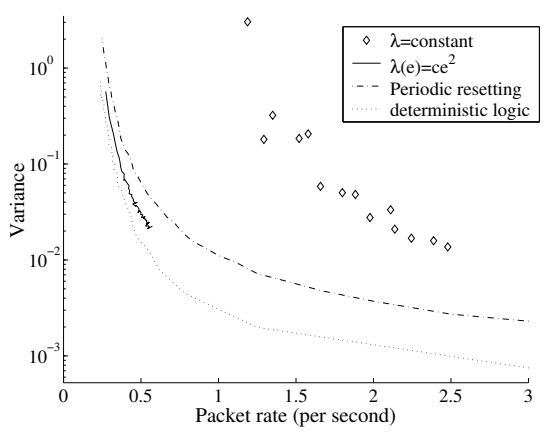

Fig. 2. Communication rate versus variance of the estimation error for different communication logics.

Fig. 3 provides a comparison between deterministic and DSPP logics. The deterministic logics have communication 
TABLE I

COMMUNICATION RATE VERSUS VARIANCE OF THE ESTIMATION AND TRACKING ERRORS

\begin{tabular}{|l|c|c|c|c|}
\hline Logics & Parameters & Comm. rate & Estim. error var. & Tracking error var. \\
\hline Deterministic & $S(e):=e \cdot P e \leq 1.15 \times 10^{-4}$ & 1.01 & $0.14 \times 10^{-4}$ & $1.1 \times 10^{-4}$ \\
\hline DSPP & $\lambda(e):=2 \frac{e \cdot P e}{1.15 \times 10^{-4}}$ & 0.95 & $0.33 \times 10^{-4}$ & $2.6 \times 10^{-4}$ \\
\hline Periodic & period $=1$ & 1 & $0.63 \times 10^{-4}$ & $3.2 \times 10^{-4}$ \\
\hline
\end{tabular}

index of form $S(e):=\frac{e^{2}}{\Delta} \leq 1$, and the different points on the curve are generated by changing $\Delta$. The DSPP logics have intensities of the form $\lambda(e)=\left(\frac{e^{2}}{\Delta}\right)^{k}$, where $\Delta$ is a positive parameter and $k \in\{1,2,3,4,5\}$. For large $k, \lambda(e)$ essentially provides a barrier at $e^{2}=\Delta$, which acts as the bound in the deterministic logics. It is therefore not surprising to see that as $k$ increases, the DSPP logics converge to the deterministic logics.

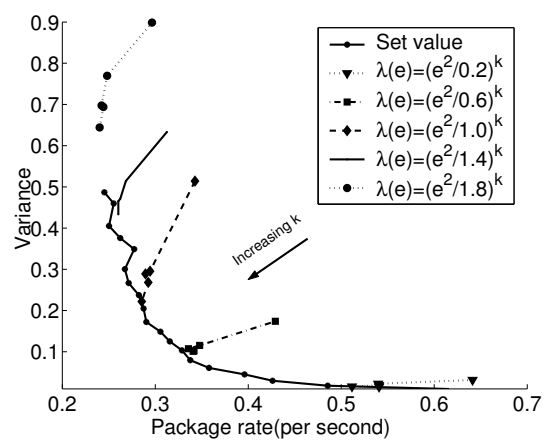

Fig. 3. Communication rate versus variance of the estimation error for deterministic and polynomial-intensity DSPP logics.

\section{CONCLUSION AND FUTURE WORK}

Deterministic and stochastic communication logics are proposed to determine when local controllers should communicate in a distributed control architecture. Using tools from jump diffusion process and the Dynkin's equation, we investigated conditions under which these logics guarantee boundedness as well as the trade-off between the amount of information exchanged and the performance achieved. Monte Carlo simulations show that these communication logics can save communication resources over periodic schemes.

Future work includes studying the impact of modelling errors on the system's performance as well as the impact of a non-ideal network that exhibits delays and may drop packets. Another topic for future research is the design of communication logics that are optimal in the sense that they minimize network traffic for a given desired variance of the estimation errors.

\section{APPENDIX}

Proof: [Lemma 1] Suppose $\mathbf{x}$ has distribution $\mu(\mathbf{x})$. For every $\delta>0$, the following inequalities hold

$$
\mathrm{E}\left[\mathbf{x}^{k}\right] \geq \int_{x \geq \delta} x^{k} d \mu(x)
$$

$$
\begin{aligned}
& \geq \delta^{\ell} \int_{x \geq \delta} x^{k-\ell} d \mu(x) \\
& =\delta^{\ell}\left(\int_{x \geq 0} x^{k-\ell} d \mu(x)-\int_{x<\delta} x^{k-\ell} d \mu(x)\right) \\
& \geq \delta^{\ell}\left(\mathrm{E}\left[\mathbf{x}^{k-\ell}\right]-\delta^{k-\ell}\right) .
\end{aligned}
$$

\section{ACKNOWLEDGMENTS}

The authors thank Professor Guillaume Bonnet for insightful discussions.

\section{REFERENCES}

[1] J. M. Fowler and R. D'Andrea, "Distributed control of close formation flight," in Proc. of the 4lth Conf. on Decision and Contr., vol. 3, Dec. 2002, pp. 2972-2977.

[2] W. S. Wong and R. W. Brockett, "Systems with finite communication bandwidth-part I: State estimation problems," IEEE Trans. on Automat. Contr., vol. 42, no. 9, Sept. 1997.

[3] - "Systems with finite communication bandwidth-II: Stabilization with limited information feedback," IEEE Trans. on Automat. Contr, vol. 44, no. 5, May 1999.

[4] G. N. Nair and R. J. Evans, "Communication-limited stabilization of linear systems," in Proc. of the 39th Conf. on Decision and Contr., vol. 1, Dec. 2000, pp. 1005-1010.

[5] S. Tatikonda, "Control under communication constrains," Ph.D. dissertation, MIT, Cambridge, MA, 2000.

[6] I. R. Petersen and A. V. Savkin, "Multi-rate stabilization of multivariable discrete-time linear systems via a limited capacity communication channel," in Proc. of the 40th Conf. on Decision and Contr., vol. 1, Dec. 2001, pp. 304-309.

[7] D. Liberzon, "A note on stabilization of linear systems with limited information," in Proc. of the 41th Conf. on Decision and Contr., vol. 1, Dec. 2002, pp. 836-841.

[8] J. P. Hespanha, A. Ortega, and L. Vasudevan, "Towards the control of linear systems with minimum bit-rate," in Proc. of the Int. Symp. on the Mathematical Theory of Networks and Syst., Aug. 2002.

[9] J. K. Yook, D. M. Tilbury, , and N. R. Soparkar, "Trading computation for bandwidth: Reducing communication in distributed control systems using state estimators," IEEE Trans. Contr. Syst. Technol., vol. 10 , no. 4, pp. 503-518, July 2002.

[10] D. R. Cox, "Some statistical methods connected with series of events," Journal of the Royal Statistical Society, vol. 17, no. 2, pp. 129-164, 1955.

[11] D. Revuz and M. Yor, Continuous Martingles and Brownian Motion, 3rd ed. Berlin: Springer, 1999, vol. 293.

[12] H. Kushner, Heavy traffic analysis of controlled queueing and communication networks, ser. Applications of Mathematics. Berlin: Springer, 2001, vol. 47.

[13] B. Oksendal, Stochastic Differential Equations: An Introduction with Applications. Berlin: Springer, 2000.

[14] Y. Xu and J. P. Hespanha, "Communication logics for networked control systems," CCEC in UC Santa Barbara, Tech. Rep., Sept. 2003.

[15] Z. Schuss, Theory and Applications of Stochastic Differential Equations, ser. Probability and mathematical statistics. Willey, 1980.

[16] P. Glasserman and N. Merener., "Numerical solution of jumpdiffusion LIBOR market models," Finance and Stochastics, vol. 7, pp. 1-27, 2003. 